\title{
CONTINUOUS INDUSTRIAL-SCALE MICROWAVE-ASSISTED EXTRACTION OF HIGH-VALUE INGREDIENTS FROM NATURAL BIOMASS
}

\author{
M. Radoiu, S. Splinter, T. Popek \\ Radient Technologies Inc., 8223 Roper Road, T6E 6S4, Edmonton, Alberta, Canada \\ mradoiu@radientinc.com
}

Keywords: industrial microwaves, biomass extraction, scale-up, continuous flow processing

\begin{abstract}
An innovative technology for the continuous extraction of bioactive compounds from a wide range of biological materials has been developed, scaled up and successfully demonstrated at commercially relevant scales. The technology, known as MAP ${ }^{\mathrm{TM}}$, or Microwave-Assisted Process, robustly transfers from laboratory to continuous, industrial scale operation. In wide-ranging trials, MAP ${ }^{\mathrm{TM}}$ has comprehensively demonstrated its ability to outperform many KPIs of conventional extraction processes, while offering biomass throughput, product consistency and low operational costs not attainable by other emerging technologies.
\end{abstract}

\section{Introduction}

Plant extracts have been long used by traditional healers for providing health benefits and are nowadays suitable ingredients to produce formulated health products and nutraceuticals. Traditional (conventional) methods of extraction such as maceration, percolation, digestion, and preparation of decoctions and infusions are now been replaced by advanced extraction methods with a view of increased extraction efficiency and selectivity of bioactive compounds to meet increasing market demand. The exponentially increasing demand of herbal products and/or extracts for wider and safer applications, timely availability of highquality products with low cost of processing and higher yield are the needs of the growing herbal/nutraceutical medicine and food-based industries. To meet these challenges, there is an increased demand for alternative and more advanced extraction techniques. Advanced techniques use various means to enhance extraction, including for example microwaves, ultrasound waves, supercritical fluids, enzymes, pressurized liquids, pulsed electric fields, etc. [1]. However, to reduce the energy and material utilization inefficiencies and increase economic and environmental benefits, these advanced technologies must be more efficient than conventional extraction methods.

To enable the development of such technologies, a closer approach via process intensification and the use of microwave (MW) effects may help achieve these goals. Microwaves do not heat by the normal methods of conduction, convection and radiation from hot surfaces, but rather by direct interaction of the wave with the material being heating via dielectric loss. The resulting instant and volumetric heating can be exploited to achieve various benefits, as long as systems and equipment are designed and implemented properly. Process intensification refers to the development of novel equipment and/or methods that produce significantly higher yields or superior benefits in comparison with the existing equipment and/or methods in practice. These benefits can be realized in the form of dramatic reduction in processing times, significant improvements in product quality or quantity, decreasing the equipment size, reducing the complexity of production schemes, improving the energy efficiency, minimizing the waste production, and finally resulting in cheaper, 
safer and sustainable technologies [2]. The process intensification developments in equipment could focus on developing novel reactor design with intense mixing to promote heat- and mass-transfer while the developments in methods could focus on integrating the reaction-separation processes (minimizing process steps) with the use of alternative energy sources, including new process control techniques. For example, in the context of natural ingredients production, process intensification efforts refer to the increasing mass and heat transfer rates among the reaction products whether in extraction and/or separation and/or purification stages.

During scale-up from bench to commercial scale, a primary issue with the MW based methods is microwave penetration depth into the plant / solvent mixture, which is only a few centimetres in usual solvents. This issue can and then exclude the use of high-volume batch reactors. Because of this, the features that make the microwave-assisted process attractive (e.g. fast extraction rates, high extraction efficiency, improved energy efficiency, etc.) may not be met. Coupling microwave heating and continuous flow technology eliminates the main drawbacks of microwaves and creates a very promising way to produce high valueadded extracts since unlike the batch, the continuous flow has been demonstrated to facilitate process intensification and contribute to a safe, efficient and sustainable production. Further, if selective recovery of valuable products from the biomass can be enhanced by manipulating the unique characteristics of the microwave process intensification effects, such process can be justified from all chemical, energy and cost related aspects.

In this paper, the basis of the MAP ${ }^{\mathrm{TM}}$ technology will be described and select scale-up studies presented.

\section{Scale-up of microwave-assisted extraction: design \& validation of the MAP ${ }^{\mathrm{TM}}$ continuous-flow extractor}

For efficient continuous-flow MW extraction outcomes and scale-up, not only the MW frequency used for extraction but also the factor consideration and their linear, quadratic and interactive effects are of great value for understanding their effect on results of maximizing the compound yield while minimizing the energy loss and solvent consumption, which ultimately add to the economic value. Various factors based on the solvents, raw materials and MW parameters need to be considered for optimum extraction design - Fig. 1. With regards to MW parameters, it is important to consider that for continuous flow extraction the dielectric and thermal properties in the reaction volume are both spatially and temporally variable [3]. In addition, the variability of the properties is not the only key factor for a good coupling of the electromagnetic field with the medium. The value itself of the dielectric properties is important, since the electric field propagation and amplitude depend respectively on the real and imaginary part of the dielectric permittivity. In the special case of biomass extraction (heterogeneous reaction with solid phase) or in general when dielectric properties increase with composition or temperature, the absorption rate of microwave energy also increases, hence thermal runaway can result. Consequently, controlling heating rate and electromagnetic field homogeneity are essential for both repeatability and industrial applications.

Radient's proprietary $915 \mathrm{MHz}$ continuous-flow MAP ${ }^{\mathrm{TM}}$ extractor, Fig. 2, was designed for continuous processing of up to $200 \mathrm{~kg} / \mathrm{h}$ of biomass material. 
The reactor consists of three main components:

- The microwave generator with variable power up to $72 \mathrm{~kW}$ based on a $\mathrm{CW}$ magnetron and switch mode power supply;

- The microwave transmission line (standard WR975 waveguides) includes the isolator (circulator + water load) \& reflected power meter, automatic tuning components, and a 2-way waveguide splitter to distribute the microwave energy to the reactor (couplings);

- The microwave multimode resonant cavity, aka continuous flow reactor made of stainless steel. Within the reactor there is a mechanical agitator, designed to prevent backflow of material.

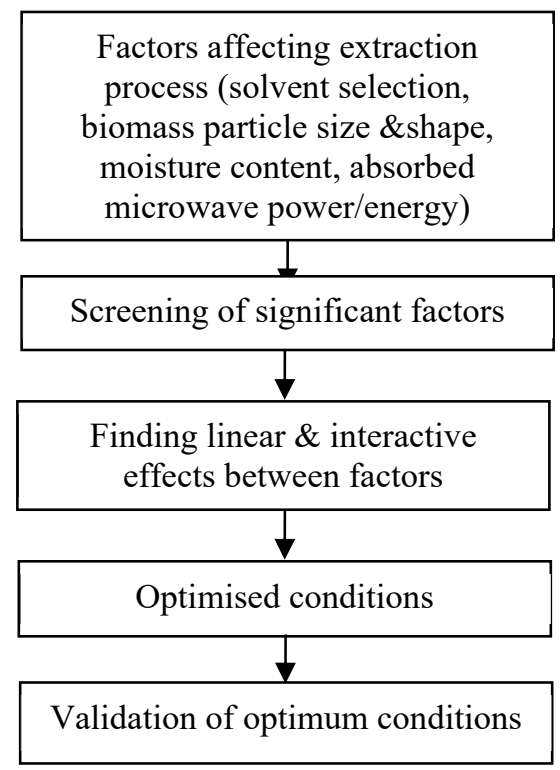

Fig. 1. Scale-up steps

Verification of the mechanical integrity of the system was confirmed by flow testing of biomass / solvent slurries. Testing and verification of the efficiency of microwave energy transfer to the extractor cavity was completed at various microwave power settings using flowing water at $870 \mathrm{~kg} / \mathrm{h}$. The microwave energy transfer to the system was verified to be $>95 \%$ in each case.

As an example of performance, continuous flow $\mathrm{MAP}^{\mathrm{TM}}$ extraction of the antioxidant SDG from flax biomass was performed using $70 \%$ ethanol / water as the solvent at two different conditions:

- $\quad 75 \mathrm{~kg} / \mathrm{h}$ flax / $5 \mathrm{~L} / \mathrm{kg}$ solvent / $15 \mathrm{~kW}$ microwave power / extractor residence time $24 \mathrm{~min}$;

- $\quad 110 \mathrm{~kg} / \mathrm{h}$ flax / $5 \mathrm{~L} / \mathrm{kg}$ solvent / $20 \mathrm{~kW}$ microwave power / extractor residence time 16 min.

The industrial-scale conditions for these runs were determined by extrapolating from optimized conditions previously obtained from batch lab-scale MAP ${ }^{\mathrm{TM}}$ experiments. The continuous flow approach eliminates the requirement for having geometric similarity 
between scales, i.e. the equipment shape and dimensions do not have to scale proportionately.

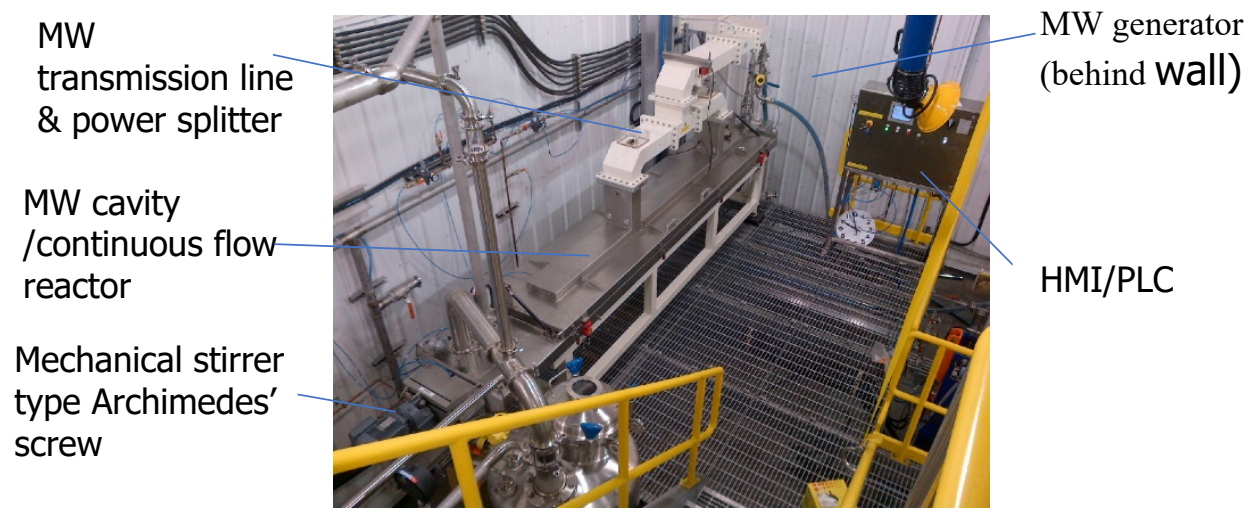

Fig. 2. Continuous-Flow Industrial-Scale MAP ${ }^{\mathrm{TM}}$ Extractor in ATEX environment

Figure 3 shows a comparison of the steady state recovery of SDG from flax obtained from lab experiments using single-stage conventional dispersed-phase mixing extraction, labscale MAP ${ }^{\mathrm{TM}}$ using the same solvent and solvent composition, and the results of the two industrial scale runs described above. From the figure, it is clear that scale-up was verified by obtaining identical results at two different flow rates and associated MAP ${ }^{\mathrm{TM}}$ energy densities. This confirmed that laboratory-scale MAP ${ }^{\mathrm{TM}}$ results can be successfully replicated at industrial scale and so verifies that the industrial-scale extractor performs as designed.

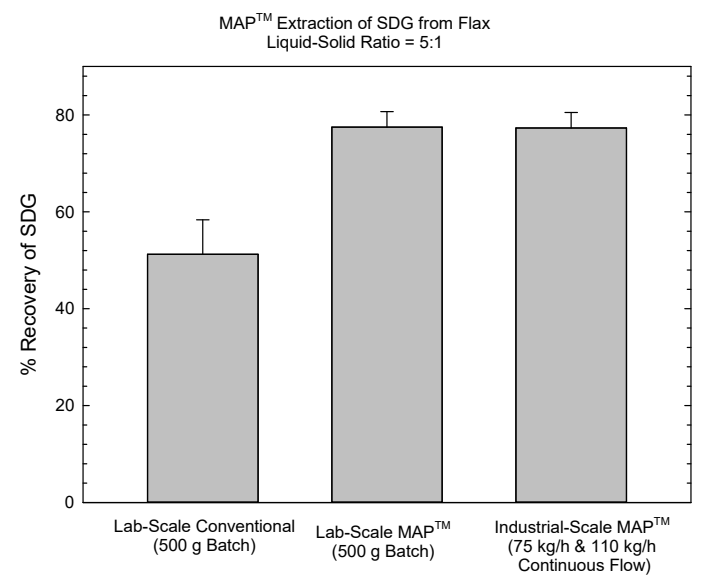

Fig. 3. Comparison of conventional extraction, lab-scale MAP ${ }^{\mathrm{TM}}$ Extraction and industrialscale MAP ${ }^{\mathrm{TM}}$ extraction of flax lignans (SDG) from flax biomass 


\section{Conclusions}

There is a necessity to explore new reactor concepts by emphasising dedicated designs that assure controllability and monitoring of the process conditions. A number of variables affect the microwave reactor output and its scale: electric field intensity, power dissipation rate and temperature of heated mixture being the most important.

It is equally important to stress that microwave technology cannot be implemented in straightforward manner to any heated industrial process. The development of microwave equipment/installations needs to be studied individually for each system in order to achieve the desired benefits. Moreover, given the interest of a chemical industry in transition from batch to continuous processing, the microwave flow reactors become increasingly interesting to the industry.

Within the ISM band (Industrial, Scientific and Medical), $915 \mathrm{MHz}$ is a frequency that presents a lot of interest for scaling up processes developed using $2.45 \mathrm{GHz}$. Industrial microwave generators operated at $915 \mathrm{MHz}$ are available up to $120 \mathrm{~kW} / \mathrm{unit}$ compared to 6 $\mathrm{kW}$ at $2.45 \mathrm{GHz}$, meaning that the quantities that can be processed are much larger using $915 \mathrm{MHz}$ equipment. In other words, a microwave process requiring for example $60 \mathrm{~kW}$, can be designed around an equipment in which the microwave power is delivered using only 1off microwave generator at $915 \mathrm{MHz}$ or 10 off generators operating at $2.45 \mathrm{GHz}$. From a viewpoint of capital investment, but also of operation and complexity of the reactor, the 915 $\mathrm{MHz}$ is by far, the more economical.

Our work was focused on investigating the scaling-up of $915 \mathrm{MHz}$ continuous flow process to industrial scales. Based on laboratory experiments and their optimisation, the continuous flow extractor for industrial biomass extraction using MAP ${ }^{\mathrm{TM}}$ was built and successfully demonstrated on various solvent - biomass matrices. The extractor comes with several benefits, including significantly increased flexibility with respect to operation:

- The contact time between the biomass and solvent before, during and after microwave treatment can be adjusted much more easily;

- It is possible to precisely control biomass residence time in the microwave zone and - if desired - separate the biomass from the solvent very quickly after treatment or continue contact for any length of time at any temperature, depending on the desired outcome.

The extractor is also easily scalable. The continuous flow approach eliminates the requirement for having geometric similarity between scales (i.e. the equipment shape and dimensions do not have to scale proportionately). Classically, even geometric similarity does not ensure thermal similarity in scaled systems - for example, heat transfer is an interface-controlled process and so the surface area relative to the volume is critical. As volumetric scale increases, the area relative to the volume decreases and the overall efficiency of heat transfer can decline considerably. There is no thermal inertia with microwaves, on the other hand. Since penetration depth is not an issue with the continuous flow design, the energy is deposited uniformly throughout the mixture resulting in rapid energy transfer and direct dielectric heating - hence the thermal inertia inherent to classical methods is not an issue.

\section{References}

1. Belwal, T., Ezzat, S.M., Rastrelli, L., Bhatt, I.D., Daglia, M., Baldi, A., Devkota, H.P., Orhan, I.E., Patra, J.K., Das, G., Anandharamakrishnan, C., Gomez-Gomez, L., Nabavi, S.F., Nabavi, S.M., Atanasov, A.G., Trends in Analytical Chemistry, 2018, 100, 82-102.

2. Stankiewicz, A.I., Moulijn, J.E., Chem. Eng. Process, 2010, 96, 22-34.

3. Estel, L., Poux, M., Benamara, N., Polaert, I., Chem. Eng. Process, 2017, 113, 5664. 